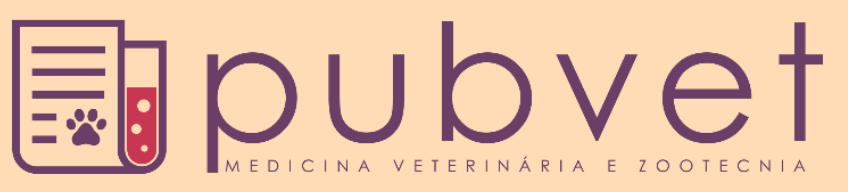

https://doi.org/10.31533/pubvet.v12n11a206.1-4

\title{
Desenvolvimento de sistema de vídeo para microcirurgia
}

\author{
Paulo Cesar Silva ${ }^{1 *} \bullet$, Alberto Schanaider ${ }^{2}$, José Eduardo Ferreira Manso ${ }^{3} \bullet$, José Marcus \\ Raso Eulálio ${ }^{\bullet}$, Manoel Luiz Ferreira ${ }^{\circ}$, Ricardo Luis Vanz $^{6} \bullet$, Gabriel Neves dos Santos \\ Mosqueira Gomes ${ }^{7} 0$
}

\begin{abstract}
${ }^{1}$ Professor Adjunto, Médico Veterinário, Universidade Federal do Rio de Janeiro, Faculdade de Medicina, Departamento de Cirurgia (Rio de Janeiro-RJ Brasil) ${ }^{2}$ ProfessorTitular, Universidade Federal do Rio de Janeiro, Faculdade de Medicina, Departamento de Cirurgia (Rio de Janeiro-RJ Brasil) ${ }^{3}$ Professor Associado, Universidade Federal do Rio de Janeiro Faculdade de Medicina, Departamento de Cirurgia (Rio de Janeiro- RJ Brasil) ${ }^{4}$ Professor Adjunto, Universidade Federal do Rio de Janeiro, Faculdade de Medicina, Departamento de Cirurgia, (Rio de Janeiro- RJ Brasil) ${ }^{5}$ Professor Adjunto, Médico Veterinário, Universidade Federal do Rio de Janeiro, Faculdade de Medicina, Departamento de Cirurgia (Rio de Janeiro-RJ Brasil) ${ }^{6}$ Mestrando, Programa de Pós-Graduação Ciências Cirúrgicas, Universidade Federal do Rio de Janeiro, Faculdade de Medicina (Rio de Janeiro-RJ Brasil) ${ }^{7}$ Graduando, Curso de Medicina, Universidade Federal do Rio de Janeiro (Rio de Janeiro- RJ Brasil)

*Autor para correspondência, E-mail:psolvis@gmail.com
\end{abstract}

RESUMO. A microcirurgia é uma abordagem cirúrgica que permite a realizaçãode atos operatórios em segmentos anatômicos milimétricos. Utiliza basicamente o microscópio cirúrgico. Idealizamos a montagem de um sistema de vídeo, que tem como objetivo a aplicação em procedimentos operatórios microcirúrgicos. Os resultados indicam a possibilidade da utilização deste sistema de vídeo em estruturas de dimensões reduzidas.

Palavras chave: animais, sistema de suporte, vídeo microcirurgia

\section{Development of video system for microsurgery}

ABSTRACT. Microsurgery is a surgical approach that allows the performance of operative acts in anatomical segments millimetric. Uses basically the surgical microscope. We have idealized the assembly of a video system, which has as its objective the application in surgical microsurgical procedures. The results indicate the possibility of using this video system in small structures.

Keywords: animals, support system, video microsurgery

\section{Desarrollo del sistema de vídeo para microcirugía}

RESUMEN. La microcirugía es un abordaje quirúrgico que permite la realización de actos operativos en segmentos anatómicos milimétricos. Utiliza básicamente el microscopio quirúrgico. Idealizamos el montaje de un sistema de video, que tiene como objetivo la aplicación en procedimientos operatorios microquirúrgicos. Los resultados indican la posibilidad de utilizar este sistema de vídeo en estructuras de dimensiones reducidas.

Palabras clave: animales, sistema de suporte, vídeo microcirugía

\section{Introdução}

A evolução tecnológica de imagens, atinge os diversos campos da atividade biológica e não é diferente na aplicação de técnicas na área cirúrgica. Com a inclusão das lupas de aumento de $1 \mathrm{X}$ a $5 \mathrm{X}$, no século XIX e XX e a adoção do microscópio estereoscópico na cirurgia no início da década de 60 do século passado, possibilitou o acesso as estruturas anatômicas mensuradas em escala milimétrica, permitindo a realização de procedimentos cirúrgicos no campo microscópico (Daniller \& Strauch, 1976).

O advento da eletrônica, aplicada aos equipamentos ópticos, possibilitou o aprimoramento da qualidade da imagem gerada na forma de vídeo. Com esta abordagem tecnológica, 
surgiram estudos de adaptação de câmeras endoscópicas e vídeolaparoscópicas para a realização de procedimentos microcirúrgicos. Desta forma aventaram a possibilidade de um sistema de visualização em cirurgia, com o uso conjunto do monitor e câmera de endoscópio, apoiados em um suporte, tendo como inconveniente a obtenção de imagem invertida, coma dificuldade inicial de coordenação da visãomão e da profundidade de campo (Ramakrishnan et al., 1997).Apesar do avanço tecnológico, existem dificuldades na obtenção de equipamentos de vídeo específicos para esta finalidade que possam ser utilizados na rotina cirúrgica.

Com o objetivo de realizar estudos utilizando técnicas cirúrgicas em estruturas anatômicas de dimensões milimétricas, idealizamos um sistema de ampliação projetado em vídeo, montado em suporte articulado, no qual se adapta câmera digital manual, com zoom óptico e digital variando de $60 \mathrm{X}$ a $1800 \mathrm{X}$. A imagem captada, projetada no monitor de televisão de alta definição, de 14 polegadas. Esta pesquisa verifica a possibilidade de montagem e uso de equipamento digital de imagem, para a aplicação em microcirurgia.

\section{Material e métodos}

O equipamento é constituído de uma haste de fixação da câmera de vídeo, composta por tubo de policloreto de vinila (PVC), rígido, de diâmetro de três-quarto $(25 \mathrm{~mm})$, com comprimento de $1,00 \mathrm{~m}$. Nas duas extremidades deste tubo, são confeccionadas roscas, uma para encaixe de um segmento tubular rosqueável (luva) de $4 \mathrm{~cm}$, no qual é afixado parafuso de 5 $\mathrm{mm}$ de diâmetro, para posicionara câmera digital por meio do seu encaixe para suporte do tripé. A outra extremidade, rosqueada a um conjunto constituído por uma peça em $\mathrm{T}$ e um tubo de $30 \mathrm{~cm}$. Este conjunto é posicionado por meio de braçadeira metálica regulável por parafuso sem-fim, ao tubo que forma o pedestal metálico, o qual sustenta o conjunto câmera de vídeo-haste de fixação (Figura 1).

A câmera de vídeo digital é um modelo manual, com zoom óptico de até $70 \mathrm{X}$ estendido e digital de $1800 \mathrm{X}$, estabilizador de imagem, abertura de lente $\mathrm{f} / 1.8$, ajustamento automático de foco, distância focal mínimo $1,8 \mathrm{~mm}$ e máximo de $108 \mathrm{~mm}$. A resolução fotográfica (megapixels) padrão de disposição gráfica de $640 \times 480$, formato de foto, com o método de compressão de imagem fotográfica $44 \mathrm{~mm} \sim 2640 \mathrm{~mm}$ (4:3). A resolução de vídeo, no formato de vídeo imagem de definição normal, com formato de compressão de áudio de $39 \mathrm{~mm} \sim 2340 \mathrm{~mm}$ (16:9). Mídia de gravação em cartão de memória 4 gigabytes, tempo de gravação $2 \mathrm{~h} 50 \mathrm{~min}$, iluminação padrão de 3 lux (Figura 2).

O monitor de tv com tela de 14 polegadas, alta definição, com ângulo de visão de $90^{\circ}$. ajuste de formato da tela, resolução $1366 \times 768$, brilho de $200 \mathrm{~cd} / \mathrm{m}^{2}$, contraste de 500:1, congelamento de imagem. Conexões com entrada padrão de disposição gráfica computador-monitor e sistemas de cores vermelho, verde e azul. Entrada de áudio do computador / entradas de áudio e vídeo e porta universal. Peso bruto de $931 \mathrm{~g}$

$\mathrm{O}$ funcionamento do equipamento ocorre com a câmera de vídeo, acoplada a haste de fixação, giratória. A objetiva focaliza a área correspondente ao campo operatório, localizado sobre a mesa cirúrgica. Uma vez obtida a imagem, são realizados os ajustes de posicionamento da câmara. Reproduzida a imagem no monitor e através da alavanca de zoom da câmara, se consegue controlar a ampliação da imagem. (Figura 3).

Na moldura da tela do monitor, foi estabelecido um padrão de escala de mensuração em centímetros, uma horizontal e outra vertical, com pontos coloridos, equidistantes de 10 centímetros, o que permite determinar de modo bidimensional a extensão da estrutura ampliada na tela do monitor. (Figura 4).

Quando associado ao microscópico cirúrgico, o suporte da câmera, é posicionado ao lado da mesa operatória, de modo que permite a visibilização simultânea da área correspondente a operatória, tanto através do microscópio cirúrgico -visão vertical, quanto no monitor via câmera digital - visão horizontal.

\section{Resultados}

O material utilizado para a confecção do suporte da câmera, constituído de estruturas tubulares de PVC, demonstra ser adequado devido à fácil aquisição comercial, leveza do material, facilidade de montagem, possibilidade de montagem modal e de adequar a diferentes necessidades no ambiente cirúrgico, resistência aos agentes químicos de desinfecção. 


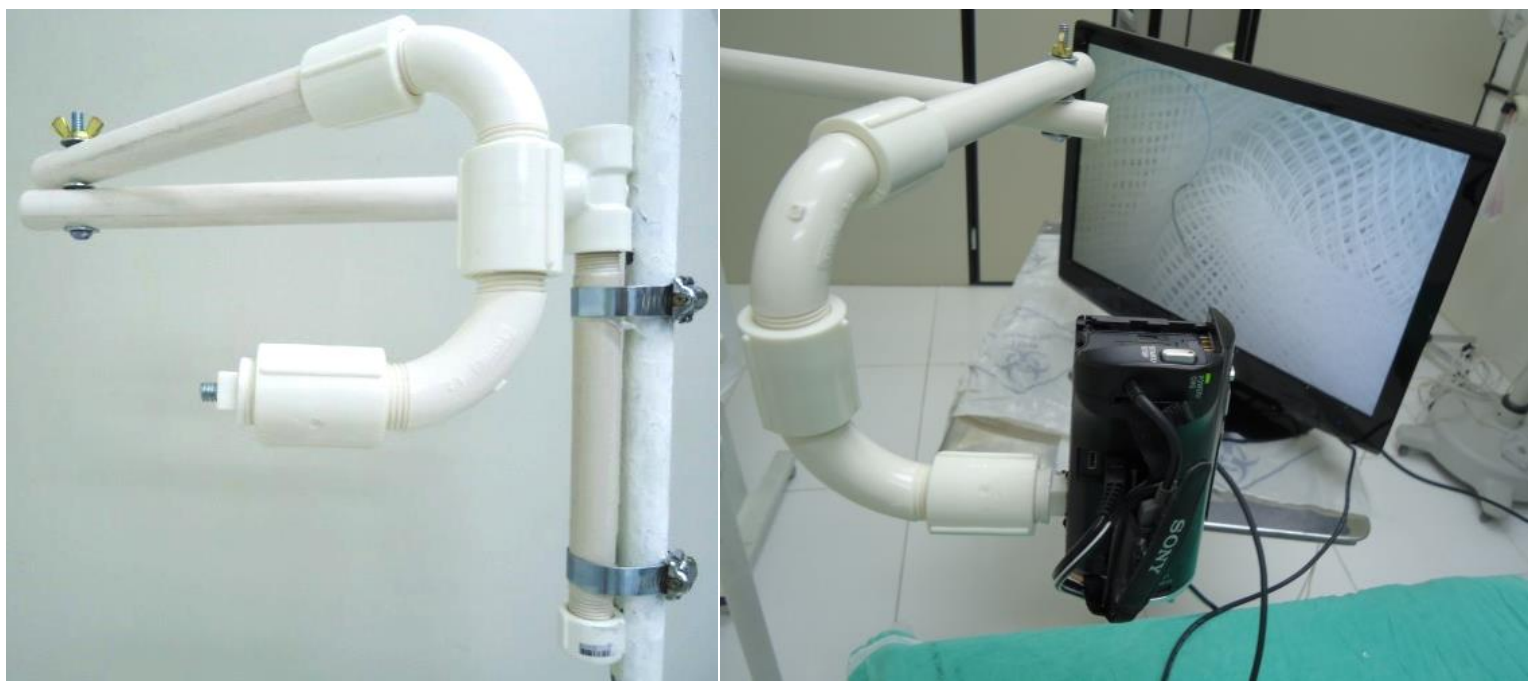

Figura 1. Haste de fixação da câmera.

Figura 2.Câmera de vídeo afixada a hastede vídeo.

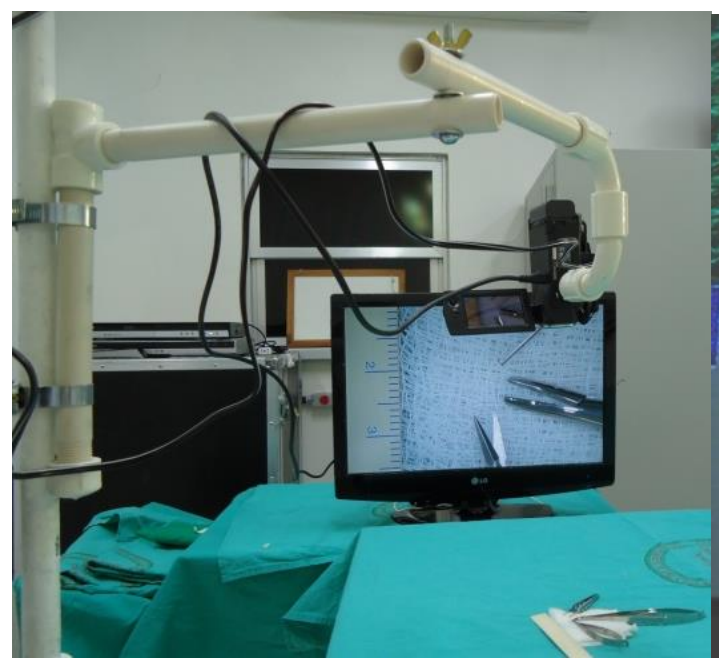

Figura 3. Monitor acoplado a câmera.

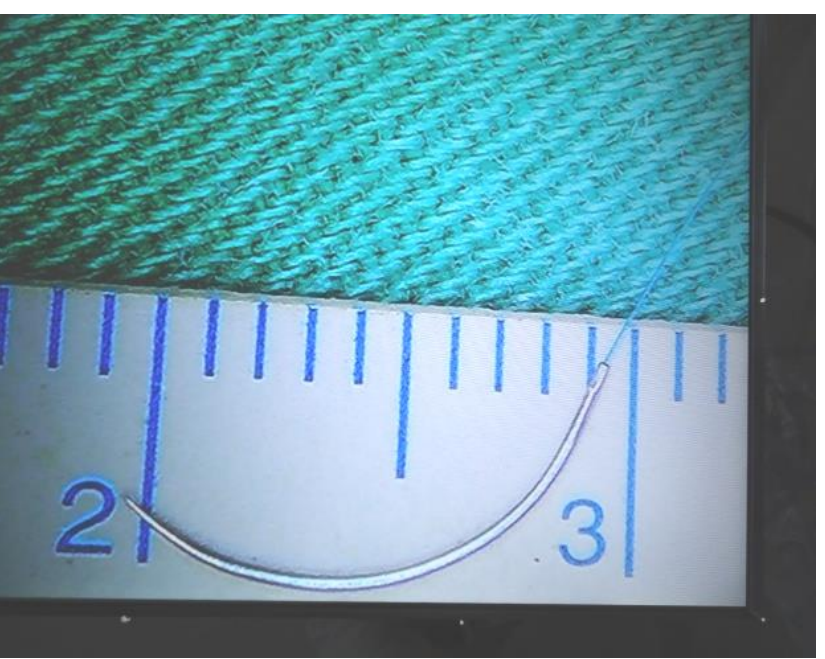

Figura 4. Moldura da tela com marcaçãode vídeo, com projeção de imagem da escala de $10 \mathrm{~cm}$ entre os pontos.
A câmera de vídeo produz imagens da área correspondente ao campo operatório de modo ampliado, a distância focal de $1,8 \mathrm{~mm}$ a $180 \mathrm{~mm}$, permite uma adequada profundidade de campo, abaixo e acima do plano focal. Outra característica é o ajuste automático do foco, o que permite a nitidez da estrutura focalizada de modo constante. Apesar de possuir iluminação própria, se utiliza a externa do foco cirúrgico de teto, de modo eficaz. A gravação das imagens em cartão de memória alojado na câmera permite a manutenção de arquivo de vídeo dos procedimentos, com possibilidades de múltiplas aplicações. $\mathrm{O}$ fato de ser um equipamento, de uso comum e de aquisição em comércio simples, favorece a aquisição e a adequação para a produção de material a ser aplicado no ensino e na pesquisa científica.

O monitor, de 14 polegadas, apresenta resolução, contraste e brilho adequados. A resolução apropriada, evita a distorção da imagem.
A coloração da área focalizada pode ser variada, mais clara ou mais escura, de acordo com a intensidade luminosa, oriunda de fonte externa. Não há dificuldades nas manobras simulatórias em relação a ser a imagem bi ou tri-dimensional, a alta definição da tela, elimina esta questão. Permite que o operador, o assistente e demais membros da equipe assistam simultaneamente a imagem correspondente ao campo operatório.

\section{Discussão}

O desenvolvimento de um sistema de vídeo para a realização de procedimentos microcirúrgicos, apesar do avanço da tecnologia de obtenção e de ampliação de imagens, ainda não está plenamente estabelecido, com uma multiplicidade de formas de uso tanto de equipamentos quanto de objetivos, conforme atesta a literatura. 
Alguns autores descrevem o uso de câmeras de endoscópios para a realização de procedimentos microcirúrgicos, comparando com o microscópio cirúrgico, descrevendo o potencial da câmera para estes procedimentos (Medot et al., 1999; Ramakrishnan et al., 1997). Estes estudos já confirmavam as possibilidades deste avanço, além da tecnologia binocular do microscópio cirúrgico, o que indica a multifuncionalidade do vídeo, conforme constatamos na realização deste projeto.

Há sistemas de vídeo idealizados para visibilização do campo operatório, usado apenas para ensino e arquivamento de imagens (Frykman et al., 2013). A aplicabilidade do sistema câmera de vídeo-monitor, no ensino com demonstração ou tutorizado; no aprendizado dirigido ou autônomo e na pesquisa realizada em estruturas milimétricas, se confirma com os resultados atualmente obtidos. A possibilidade de se utilizara tecnologia de vídeomicroscópio junto com o monitor de tv, aplicado a procedimentos microcirúrgicos simples, demonstrou ser limitado pela visão bi dimensional (Frykman et al., 2013).Com o uso de câmara de alta definição, a questão de dimensionalidade, fica ultrapassada, pois além da definição de imagem, há o aumento da profundidade campo e o auto ajuste do foco, estes fatores contribuem para que não ocorra o efeito acima exposto.

A análise das diversas formas de ampliação de imagens para aplicação em microcirurgia, descrita, com duas abordagens interessando o uso de câmeras de vídeo. Uma descreve a acoplagem diretamente ao microscópio para projeção em monitor e outra utiliza câmeras de vídeo industrial conectada a monitor tri-dimensional, sendo esta tecnologia indicada para procedimentos minimamente invasivos (Akbari et al., 2012). Outros autores com o objetivo de substituir o uso de lupas desenvolveram vídeomicroscópicos, que projetam imagem em telas, porém com aumento máximo de 16x,mas não sendo utilizados em procedimentos microcirúrgicos (Southern et al., 2001).A ampliação da imagem obtida pela câmera utilizada neste projeto, com aumento de até $20 \mathrm{X}$, permite a visibilização das áreas a serem tratadas sem dificuldades de nitidez e focalização, o que facilita a manipulação de estruturas milimétricas.

Um aspecto a ser destacado é o fato do suporte, montado com material de custo reduzido, resistente e apropriado para montagem estrutural, com capacidade de ser modelado em diversas disposições, propiciar a confecção da haste de sustentação da câmera de vídeo. Isto permite o desenvolvimento de um sistema de câmera de vídeo-monitor com equipamentos e materiais de fácil aquisição comercial, porém com qualidade tecnológica em termos de imagem.

Com os atuais recursos técnicos, no vídeo, no monitor, na ampliação, na qualidade de imagem e de acordo com os resultados aqui alcançados, indicam a possibilidade da realização plena de procedimentos micro operatórios ao menos na área de pesquisa experimental cirúrgica.

\section{Conclusão}

O sistema de vídeo-monitor proposto, conforme o estudo realizado indica a possibilidade na aplicação em procedimentos microcirúrgicos.

\section{Referências}

Akbari, G., Prabhuji, M., \& Lavanya, R. 2012. Microsurgery: a clinical philosophy for surgical craftsmanship. E-Journal of Dentistry, 2(3), 46-50.

Daniller, A. I., \& Strauch, B. 1976. Symposium on Microsurgery, St Louis, USA.

Frykman, P. K., Duel, B. P., Gangi, A., Williams, J. A., Berci, G., \& Freedman, A. L. 2013. Evaluation of a video telescopic operating microscope (VITOM) for pediatric surgery and urology: a preliminary report. Journal of Laparoendoscopic \& Advanced Surgical Techniques, 23(7), 639-643.

Medot, M., Nelissen, X., Heymans, O., Adant, J. P., \& Fissette, J. 1999. Video-microsurgery: a new tool in microsurgery. British Journal of Plastic Surgery, 52(2), 92-96.

Ramakrishnan, V. V., Villafane, O., \& Southern, S. 1997. Video microsurgery: a substitute for the operating microscope? British Journal of Plastic Surgery, 50(4), 294-296.

Southern, S. J., Ramakrishnan, V., Villofane, O., Watt, D. A. L., \& Sharpe, D. T. 2001. Video microsurgery: early experience with an alternative operating magnification system. Microsurgery, 21(2), 63-69.

Recebido:7 Setembro, 2018

Aprovado:30 Setembro, 2018.

Publicado: 21 Novembro, 2018.

Licenciamento: Este artigo é publicado na modalidade Acesso Aberto sob a licença Creative Commons Atribuição 4.0 (CC-BY 4.0), a qual permite uso irrestrito, distribuição, reprodução em qualquer meio, desde que o autor e a fonte sejam devidamente creditados. 